\title{
Aplicativo mentalpro para auxílio na suspeição de transtornos mentais na Atenção
}

\section{Básica em Saúde}

\author{
Mentalpro application for aid in suspecting mental disorders in Basic Health Care \\ Aplicaión mentalpro para la ayuda em la sospecha de transtornos mentales em la Attención Básica \\ de Salud
}

Recebido: 20/05/2021 | Revisado: 28/05/2021 | Aceito: 01/06/2021 | Publicado: 15/06/2021

\author{
Jorge Luiz Lima da Silva \\ ORCID: https://orcid.org/0000-0002-2370-6343 \\ Universidade Federal Fluminense, Brasil \\ E-mail: jorgeluizlima@gmail.com \\ Igor Barreto Meirelles \\ ORCID: https://orcid.org/0000-0001-9692-6032 \\ Universidade Federal Fluminense, Brasil \\ E-mail: igorbm@id.uff.br \\ Gabriella Filippini Silva Ramos \\ ORCID: https://orcid.org/0000-0002-2570-0498 \\ Universidade Federal Fluminense, Brasil \\ E-mail: gfilippini@id.uff.br \\ Larissa Murta Abreu \\ ORCID: https://orcid.org/0000-0002-7119-4370 \\ Universidade Federal Fluminense, Brasil \\ E-mail: larissamurtaabreu9@gmail.com \\ Alexander Rivail Ruiz Martins \\ ORCID: https://orcid.org/0000-0001-6519-2291 \\ Universidade Federal Fluminense, Brasil \\ E-mail: ruizalexander@id.uff.br
}

\begin{abstract}
Resumo
Objetivo: relatar a experiência da construção de aplicativo para auxílio da suspeição de transtornos mentais comuns, para usuários da rede básica de saúde. Metodologia: trata-se de relato de experiência do passo a passo da construção de protótipo tecnológico que objetiva a construção de aplicação híbrida, desktop e mobile, com base em escalas validadas no Brasil. Resultados: o design do aplicativo foi elaborado de forma que as etapas de coleta de informações e respostas dos questionários fossem parecidas com um chat em tempo real. As correções e adequações finais, foram realizadas mediante testagens de funcionamento e demandas que surgiram durante a elaboração, até a apresentação da versão final. Conclusão: percebe-se que o aplicativo "MentalPro" poderá ser uma ferramenta útil, em ambientes como salas de espera, pré-atendimento, consultas de enfermagem ou médicas, e triagem, auxiliando os profissionais e direcionando os cuidados adequados para promoção da saúde mental. O produto é de livre acesso e se propõe a adaptações necessárias com uso junto à comunidade.
\end{abstract}

Palavras-chave: Aplicativos móveis; Saúde mental; Tecnologia da informação em saúde.

\begin{abstract}
Objective: to report the experience of building an application to help suspect common mental disorders, for users of the basic health network. Methodology: this is an experience report of the step-by-step construction of a technological prototype that aims to build a hybrid application, desktop and mobile, based on scales validated in Brazil. Results: the design of the application was designed in such a way that the steps of collecting information and answering the questionnaires were similar to a chat in real time. The final corrections and adjustments were carried out by means of functional tests and demands that arose during the elaboration, until the presentation of the final version. Conclusion: it is clear that the "MentalPro" application can be a useful tool, in environments such as waiting rooms, pre-service, nursing or medical consultations, and screening, assisting professionals and directing appropriate care to promote mental health. Is an open access product that proposes to adapt from use.
\end{abstract}

Keywords: Mobile applications; Mental health; Health information technology.

\section{Resumen}

Objetivo: reportar la experiencia de construcción de una aplicación para ayudar a sospechar trastornos mentales comunes, para usuarios de la red básica de salud. Metodología: se trata de un informe de experiencia de la construcción paso a paso de un prototipo tecnológico que tiene como objetivo construir una aplicación híbrida, de escritorio y móvil, 
basada en escalas validadas en Brasil. Resultados: el diseño de la aplicación se diseñó de tal manera que los pasos para recolectar información y contestar los cuestionarios fueran similares a un chat en tiempo real. Las correcciones y ajustes finales se realizaron mediante pruebas funcionales y demandas que surgieron durante la elaboración, hasta la presentación de la versión final. Conclusión: es evidente que la aplicación "MentalPro" puede ser una herramienta útil, en entornos como salas de espera, pre-servicio, consultas médicas o de enfermería, y cribado, asistiendo a los profesionales y dirigiendo la atención adecuada para promover la salud mental. El producto es de libre acceso y propone las adaptaciones necesarias de uso.

Palabras clave: Aplicaciones móviles; Salud mental; Tecnología de la información sanitaria.

\section{Introdução}

Os transtornos de ordem mental constituem-se um problema de saúde pública, visto que dados da Organização Mundial da Saúde (2018) apontam que cerca de 450 milhões de indivíduos sofrem de algum tipo desses transtornos. Uma em cada quatro pessoas será afetada, em algum estágio da vida. No ano de 2020, a depressão foi a maior causa de afastamento no mundo, e com a pandemia e isolamento urge a necessidade de estratégias mitigadoras.

A porta de entrada do setor saúde é a Atenção Básica (AB), onde o usuário do Sistema Único de Saúde (SUS) busca atendimento e resolutividade, embora sabe-se que o sistema carece de profissionais treinados, de recursos para identificar aspectos referentes à saúde mental e prestar orientações. O aplicativo surge como oportunidade de auxílio (ferramenta) aos profissionais e população, nesse momento de perceptível vulnerabilidade social.

Nessa lógica, a saúde mental é produto de múltiplas e complexas interações, que incluem fatores biológicos, psicológicos e sociais. Entende-se que os fatores determinantes sociais de saúde estão imbricados na maneira como se dará o desenvolvimento dos transtornos mentais, os quais podem influenciar a saúde física e mental dos indivíduos (Gaino; Souza; Cirineu \& Tulimosky, 2018).

Assim, os Transtornos Mentais Comuns (TMCs) são representados por indivíduos que, por não poderem ser convenientemente categorizados dentro das nomenclaturas existentes em saúde mental, em protocolos e outros guias de informações, encontram-se reunidas nesse grupo, dada sua proximidade diagnóstica (Maragno et al, 2006). São exemplos de TMCs as somatizações, as fobias, os transtornos do pânico, a bulimia, os transtornos depressivos, a ansiedade generalizada e o misto destes dois últimos, dentre outros, cuja classificação se torna difícil pela complexidade e similaridade de sintomas (Kendler et al, 1995).

A prevalência desse tipo de transtornos mental entre os usuários da $\mathrm{AB}$ é elevada, especialmente em países como o Brasil (Gonçalves et al, 2014). Estudos de revisão sistemática brasileiros apontam variação entre 29,6\% a 64,3\% da prevalência dos TMC (Rocha et al, 2014, Pinto et al, 2014, Menezes \& Correia, 2017). Majoritariamente, esses grupos envolvem mulheres, trabalhadores, adolescentes, gestantes e profissionais de saúde, o que desperta o interesse, para que mais investigações sejam dirigidas entre grupos diferentes (Carlotto, 2016). Como a porta de entrada no SUS é a atenção básica, representada por unidades básicas de saúde (UBS), é importante que existam estratégias de detecção precoce e manejo dos TMCs.

Segundo o Ministério da Saúde (2020), devido ao período de distanciamento social pode ocorrer deterioração de redes sociais; estresse devido a dívidas; estigmas e rejeição; raiva e agressividade; desconfiança; desenvolvimento ou recaídas de transtornos mentais. Soma-se a isso a baixa renda, crises nos países em desenvolvimento, aumento de idosos, exigências do mundo tecnológico e da reorganização do sistema de trabalho, fatores que têm contribuído para a crescente demanda, e exigências psicossociais desencadeando o incremento da prevalência de agravos de ordem mental (Lima da Silva, 2015).

O isolamento pode intensificar os sentimentos de desamparo, tédio, solidão, tristeza e reações comportamentais como alterações ou distúrbios de apetite, distúrbios do sono e conflitos interpessoais (Tavares, 2020). Tais orientações, relacionadas ao convívio em sociedade, levaram à adoção de um novo comportamento por parte da população, causando aumento de sintomas de medo, estresse, ansiedade e depressão (Ribeiro, 2020). 
Dessa forma, o acesso à informação por meio de tecnologias, incluindo-se a internet, demonstra-se fundamental durante o período de isolamento social, surgindo como estratégia relevante para enfrentamento da pandemia. Nesse cenário, o acesso à internet por meio de dispositivos móveis, como smartphones, tem exercido papel importante, como meio de busca, e obtenção de informação relacionada à saúde (Oliveira \& Alencar, 2017).

O Centro de Tecnologia de Informação Aplicada (FGVcia) da Escola de Administração de Empresas de São Paulo da Fundação Getúlio Vargas (FGV EAESP) revela que haviam 424 milhões de dispositivos digitais em uso no Brasil, no ano de 2020. Estima-se que, ao todo, são 234 milhões de smartphones sendo utilizados diariamente (FGV, 2020).

Sabe-se que o mundo vivencia intensas transformações tecnológicas, onde a velocidade e a complexidade das tecnologias avançam desde o advento da globalização. A demanda por tecnologias da informação é crescente e assume forte influência no setor da saúde (Chaves et al, 2018). No contexto da saúde e tratamento de doenças, é incontestável o papel das ciências e tecnologias (Lorenzetti; Trindade; Pires \& Ramos, 2012).

A disseminação da internet por meio dos dispositivos móveis levou ao surgimento de uma subdivisão da saúde denominada e difundida como Saúde Móvel (mHealth), entendida como a oferta de serviços médicos e/ou de Saúde Pública que utilizam tecnologias de dispositivos móveis (OMS, 2011).

Os aplicativos móveis são programas desenvolvidos especificamente para o sistema operacional que permitem a interação e navegação, utilizando dispositivo móvel (Amorim \& Bianco, 2011). Os aplicativos podem facilitar a aprendizagem individualizada, uma vez que os dispositivos portáteis são dos próprios indivíduos, e apresentam potencial para armazenar informações que podem ser visualizadas, em qualquer lugar e momento em que o usuário se encontra (UNESCO, 2014).

Os aplicativos na área da saúde vêm ganhando espaço e ajudam no diagnóstico de doenças no Brasil e no mundo (Tibes, Dias \& Zem-Mascarenhas, 2014). Com isso, este estudo objetiva relatar a experiência da construção de aplicativo para auxílio da suspeição de transtornos mentais comuns, com a pretensão de ser utilizado por usuários da rede básica de saúde. Uma vez que as tecnologias podem ser aliadas na promoção da saúde e qualidade de vida.

\section{Metodologia}

Trata-se de relato de experiência do passo a passo da construção de um protótipo tecnológico que objetiva a construção de uma aplicação híbrida, desktop e mobile. Utilizou-se como referência os questionários validados no Brasil. As dimensões das principais escalas utilizadas em saúde mental que foram inseridas na programação: a Self Reporting Questionnaire (SRQ) que avaliaTMCs na atenção básica, criada por Goldberg e Huxley (1992); a Job Stress Scale, em seu formato reduzido para avaliar o grau de estresse no trabalho; e o inventário da síndrome do esgotamento profissional, conhecida como burnout para aqueles com sinais de alto desgaste no trabalho (Mari \& Williams, 1986; Tamayo \& Tróccoli, 2009).

A utilização do SRQ na $\mathrm{AB}$, sobretudo em países em desenvolvimento, é recomendada pela OMS, à medida em que preenche critérios acerca da facilidade de uso e custo reduzido. Tal questionário é utilizado em diversos países de culturas distintas a fim de rastrear transtornos não-psicóticos (Gonçalves, Stein \& Kapczinski, 2008). Este estudo adotou para fins de classificação sujeitos não suspeitos aqueles que apresentarem escore igual ou menor a cinco respostas positivas, e suspeitos aqueles com escore igual ou acima de seis (Ludermir \& Lewis, 2003).

Foi empregada a versão resumida da Job Stress Scale adaptada ao português, o questionário foi originalmente elaborado em inglês e visa investigar a associação entre o estresse no trabalho e variados desfechos de saúde. O instrumento contém 17 questões, sendo cinco para avaliar a demanda psicológica no trabalho, seis para avaliar o grau de controle no trabalho e seis para avaliar o apoio social no ambiente de trabalho (Alves; Chor; Faerstein; Lopes \& Werneck, 2004).

Utilizou-se a versão adaptada e validada da Maslach Burnout Inventory (MBI), para avaliação da síndrome de burnout. O instrumento é composto por 22 questões com escala de frequência com cinco pontos que vai de um até cinco, que avaliam três 
dimensões: esgotamento emocional (09 afirmativas); despersonificação (05 afirmativas) e realização profissional (08 afirmativas) (Tamayo \& Trôccoli, 2002; Lima da Silva et al, 2015; Leiter \& Maslach, 2016).

Com as bases teóricas solidificadas, iniciou-se o processo de design do aplicativo, que consiste na construção da estrutura de navegação, estruturamento visual, tipografia e responsividade das telas. Utilizou-se o software web de design colaborativo Figma para construção dos mockups necessários.

$\mathrm{Na}$ etapa de desenvolvimento, optou-se por linguagens reconhecidas no desenvolvimento web de sites e aplicativos como: Hyper Text Markup Language (HTML5), JavaScript, Cascade Style Sheet (CSS3), VueJS como framework e Firebase para armazenamento de textos, títulos, mensagens de finalização e hosting da aplicação, além da utilização do plugin do Material Design Vuetify.

\section{Resultados}

O desenvolvimento do aplicativo, consistiu-se em etapas:

1: Levantamento bibliográfico do material teórico científico realizado, no primeiro ano do projeto, financiado por três meses por bolsa de iniciação tecnológica da Universidade Federal Fluminense;

2: Levantamento e avaliação de opções desenvolvimento, realizada no segundo ano, mesmo após interrupção da bolsa;

3: Levantamento das unidades estão relacionadas ao atendimento em saúde mental, realizada no segundo ano;

4: Definição e construção da estrutura do aplicativo, sendo um piloto para testes para adequação do conteúdo pesquisado, no caso, as escalas de avaliação validadas no Brasil. Dessa forma, pode-se definir os textos que seriam apresentados ao usuário, a tela de saudação inicial (fig. 1 e 2), e a melhor forma de apresentação do conteúdo selecionado. O design foi elaborado de forma que as etapas de coleta de informações e respostas dos questionários fossem parecidas com um chat, em tempo real. Dessa maneira, à medida em que o usuário seleciona uma opção o aplicativo responde exibindo a próxima escolha a ser selecionada. A Figura 2 exibe o resultado do design de saudação inicial;

5: Adequação às plataformas disponíveis em dispositivos móveis. - Após a construção da estrutura e adaptação, foi criado um produto parcial para teste com o público, onde foram convidados, por e-mail, alguns membros do grupo de estudos e pesquisa. Teste inicial buscou bugs e falhas, e foi realizado inicialmente com 15 estudantes. O feedback ocorria por preenchimento de questionário sobre o aplicativo, desenvolvido na plataforma online Google Forms. A testagem do aplicativo viabilizou a correção de erros, e o aprimoramento, por meio de ajustes;

6: Construção de site para disponibilizar dados do projeto e produto, fase ainda em construção. - Pretende-se criar um repositório anônimo de dados, ou seja, as informações coletadas são apenas sobre aspectos da saúde e qualidade de vida, para que estudantes e profissionais de saúde tenham acesso;

7: Teste do aplicativo junto a uma unidade de saúde, fase interrompida devido à pandemia, embora nova rodada de testes tenha ocorrido com cerca de 200 usuários, via nossas redes sociais de projeto de ensino e pesquisa;

8: Correções e adequações finais, ocorreram em 2021, com base na experiência de vários usuários. - Produto final entregue. Alocado em: pensu-promental.web.app/\#/, e em fase de disponibilização, nas lojas de aplicativos, onde pretende ser grátis e livre.

O fluxo de questões (fluxograma 1) foi inserido na programação, em etapas diferentes durante a interação, dessa forma, consegue-se realizar a triagem selecionando os suspeitos. Destacam-se quatro momentos, na interação durante o uso do aplicativo:

Momento um - para aqueles usuários que não trabalham e não apresentam suspeição, os quais receberão dicas de saúde e bem-estar, sendo que se apresentarem sinais de estresse moderado, serão orientados pelas recomendações anteriores, e orientações gerais para controle do estresse e ansiedade (nessa fase, se o usuário do app apresentar escores elevados a 
programação redireciona para a fase 4);

Momento dois - para aqueles que trabalham, e a programação iniciará o questionário de estresse no trabalho, após a fase um completa;

Momento três - para aqueles suspeitos de estresse moderado, os quais são encaminhados para o questionário de esgotamento profissional; após completar a fase um e dois e, por fim;

Momento quatro - onde aqueles com risco elevado e sinais de danos, descritos nas escalas / escores são orientados com auxílio do GPS, a buscarem unidade de saúde de referência no SUS (no caso, uma UBS) (Figura 3).

Entende-se que todos são potencialmente usuários do SUS, uma vez que na fase de testes do aplicativo, os estágios foram suspensos nas unidades, assim como o recurso pode ser utilizado de forma remota.

Cabe destacar que o aplicativo não possui pretensão de diagnosticar doenças, trabalha com uma autoavaliação com foco na promoção da saúde física e mental. Para que o usuário chegue a receber a recomendação de buscar atendimento na UBS, provavelmente terá uma soma de sinais e sintomas que vão além do aspecto mental / emocional, o quais repercutem na saúde global. As dicas e saúde e bem-estar são o foco do protótipo, onde a cada dica há botões de interação, e mais 20 dicas subsequentes (Figura 4). 
Fluxograma 1: etapas da incorporação de questões na programação do aplicativo MentalPro, Niterói, Rio de Janeiro, 2021.

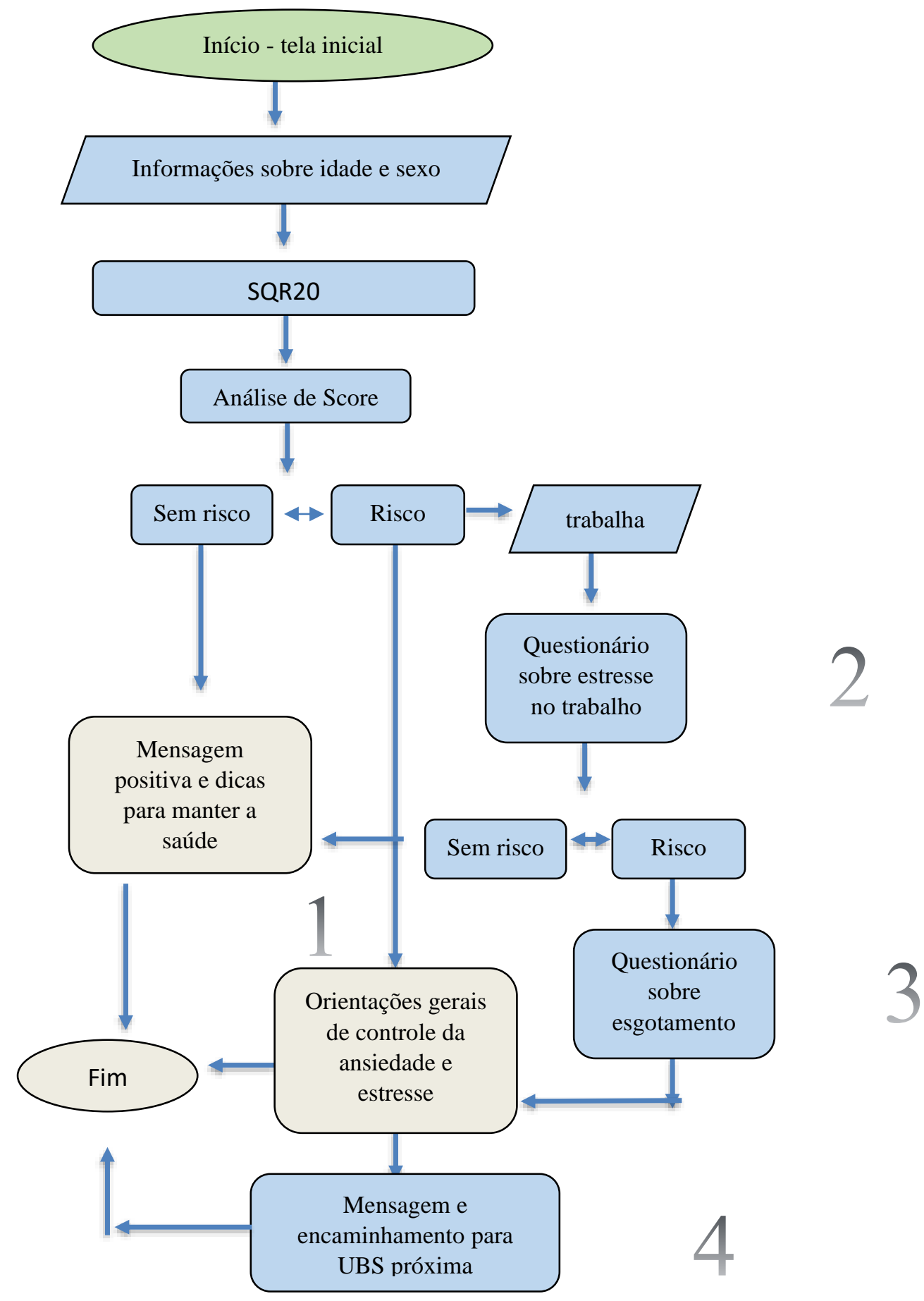

Fonte: Autores.

Para que o aplicativo fosse idealizado, o grupo contou com um bolsista do programa Pibiti (Programa Institucional de Bolsas de Iniciação em Desenvolvimento Tecnológico e Inovação), que objetiva estimular as atividades próprias do desenvolvimento tecnológico, com foco na área de inovações. Além disso, houve esforços de docentes da área de pesquisa em saúde mental, acadêmicos bolsistas e voluntários do curso de graduação em enfermagem e física, possibilitando interlocução fundamental para o desenvolvimento do projeto. 
Research, Society and Development, v. 10, n. 7, e12110716318, 2021

(CC BY 4.0) | ISSN 2525-3409 | DOI: http://dx.doi.org/10.33448/rsd-v10i7.16318

Ademais, após ajustes e testagens acerca das versões que foram adaptadas conforme demanda, o produto digital será registrado na plataforma play store. O Google Play é hoje a forma mais usada para downloads de aplicativos no Brasil, sendo a sua disponibilização neste meio fundamental para garantir a visibilidade. Nesta etapa, o desenvolvedor fornece todas as informações relacionadas ao produto digital oferecido.

Figura 1: tela de boas-vindas.

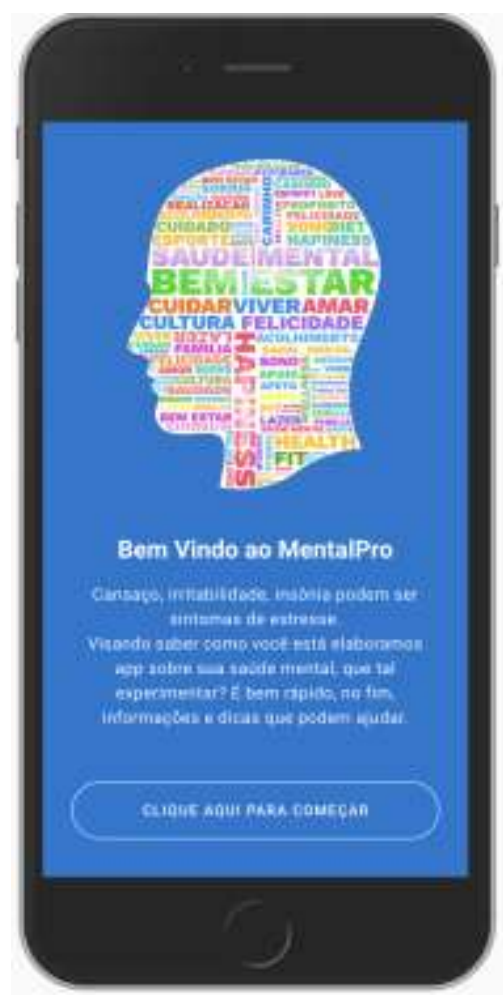

Fonte: Autores.
Figura 2: saudação inicial.

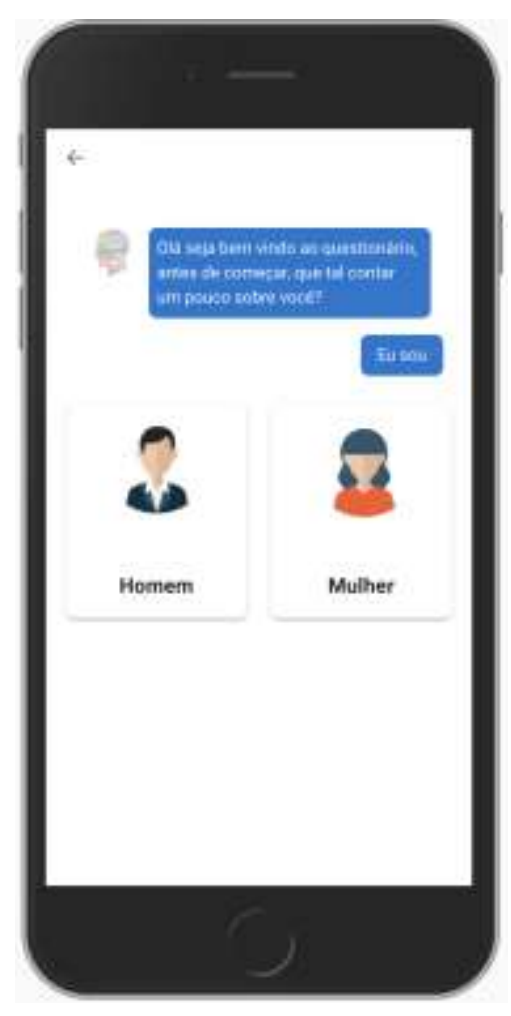

Fonte: Autores. 
Figura 3: saída para usuários com escores elevados/ suspeitos de TMC.

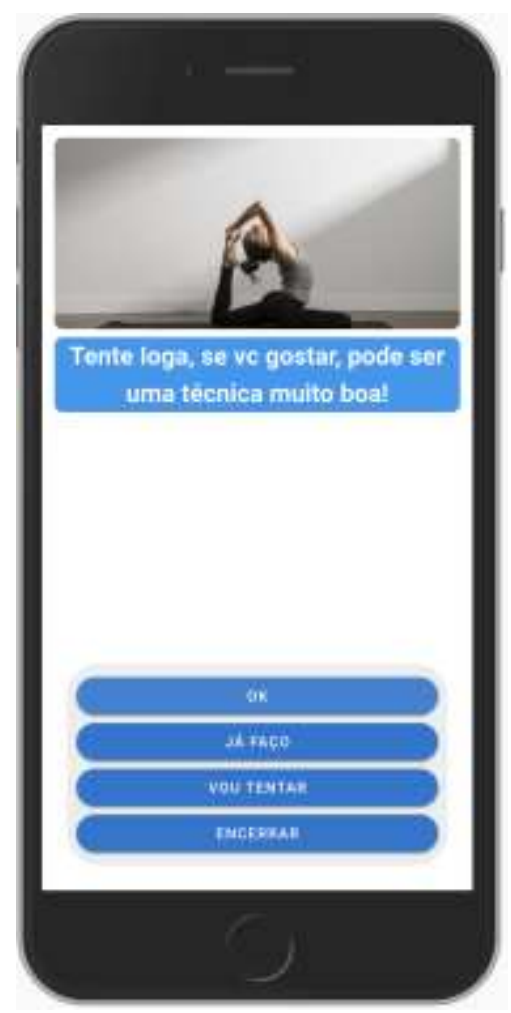

Fonte: Autores.
Figura 4: tela de dica de saúde.

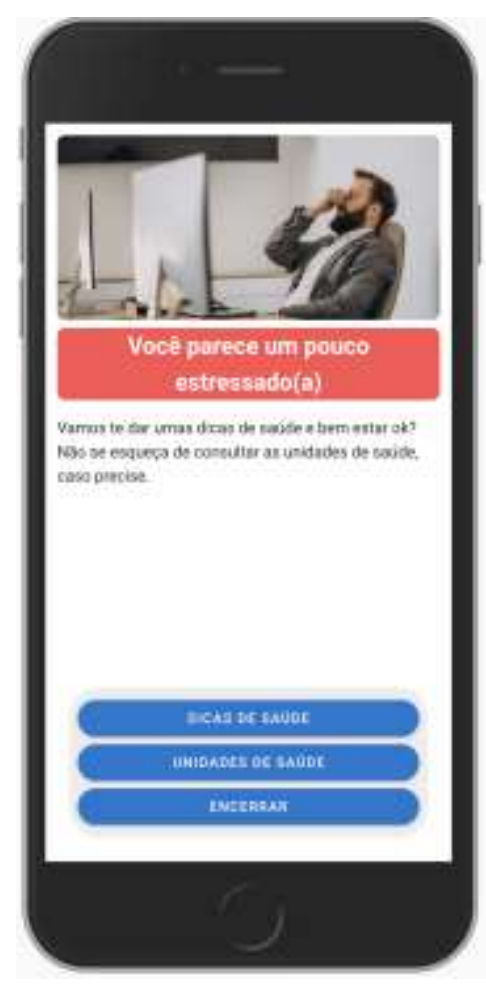

Fonte: Autores.

\section{Discussão}

Os transtornos mentais representam hoje um dos principais desafios na agenda de saúde, tanto de países desenvolvidos quanto de países em desenvolvimento, constituindo ônus importante para os serviços públicos (Lopes, 2020). Estudo sobre a carga global de doenças mostrou que, mundialmente, os transtornos mentais respondem por 32,4\% dos anos de vida vividos com incapacidade (Vigo, Thornicroft \& Atun, 2016).

O Brasil apresenta características demográficas e econômicas que têm sido apontadas de forma consistente como o pano de fundo para o aumento da incidência e persistência de transtornos mentais na população geral (GBD, 2016).

A preocupação com a saúde mental da população se intensifica, durante uma grave crise social. A pandemia da covid19 pode ser descrita como uma dessas crises, que tem se caracterizado como importante problema de saúde pública internacional (WHO, 2020).

Os aplicativos móveis configuram-se como tecnologias digitais inovadoras, frente ao cenário epidemiológico da covid19 no mundo. Além de oferecerem comodidade e benefício para população e gestores, no acesso ao conhecimento, tornam-se alternativas atrativas para a educação continuada de profissionais da saúde, por meio de estímulos visuais, táteis e auditivos (Neto, 2020).

A utilização da tecnologia para monitorar, promover cuidados e maior adesão aos tratamentos de saúde facilita a maior integração entre equipe multiprofissional e usuário/paciente. Com a exatidão de um toque na tela, tais dispositivos tornam-se aliados importantes para a educação em saúde, por meio dos aplicativos que potencializam a comunicação entre profissionais e pacientes ou usuários, encurtando distâncias e levando informações, favorecendo o processo de autocuidado, empoderamento e o acompanhamento mais seguro do processo de saúde-doença (Chaves et al., 2018). 
Em contrapartida, é necessário refletir acerca do uso abusivo de dispositivos móveis, visto que é uma preocupação constante e alarmante que impacta o componente social. Logo, sabe-se que é necessário que o uso de dispositivos obedeça ao bom senso.

O setor saúde, fortemente influenciado pelo paradigma da ciência positiva, tem sido sensível à incorporação tecnológicas do tipo material, para fins terapêuticos, utilizando os conhecimentos e produtos da informática que, no caso dos aplicativos, tem os benefícios de serem de uso aberto e livre, considerados facilitadores do processo de educação em saúde (Muraro, 2009; Mendes, 2019).

As práticas de educação em saúde em sua essência têm natureza multidisciplinar, pois agregam uma gama de profissionais com propósitos em comum, fornecendo o suporte necessário para a ampliação dos conhecimentos de saúde pelos atores envolvidos, incluindo-se os pacientes (Rocha et al., 2017).

A saúde é considerada uma área eminentemente interdisciplinar e a integração de tecnologia, por meio da utilização de aplicativos educativos, somada aos recursos humanos, certamente poderá levar à formação de profissionais mais comprometidos com a saúde e com a sua transformação (Vilela \& Mendes, 2003).

Embora este relato de construção mostre um recurso tecnológico de auxílio a estudantes e profissionais de saúde a identificar sinais e sintomas relacionados ao estresse e suas consequências (como os TMCs), de forma científica, não tem pretensões de diagnosticar doenças. Embora o desenvolvimento de apps com finalidade terapêutica é uma realidade que deve ser explorada em toda a sua amplitude, tanto pelos pacientes, quanto pela equipe multiprofissional (Heffernan et al., 2016; Geoger \& Decristoforo, 2016).

A utilização de smartphones tem refletido em um grande número de pessoas atingidas com tais práticas em saúde de maneira nunca antes imaginada na transmissão e processamento de informações (Brasil, 1992 como citado em Rocha et al, 2017, p. 3).

O produto final pretende ser grátis, de fácil acesso disponível para download em plataformas de amplo conhecimento. As orientações visam incentivar ações que promovam hábitos relacionados à qualidade de vida, com a finalidade de favorecer a saúde física e mental.

A participação de estudantes, pesquisadores e usuários do sistema de saúde fortalece o produto desenvolvido, assim como esse vínculo favorece o aperfeiçoamento, por meio de feedback, de forma contínua e interdisciplinar, uma das formas é o uso de formulários eletrônicos.

\section{Conclusão}

O aplicativo "MentalPro" poderá ser uma ferramenta útil, em ambientes como salas de espera, pré-atendimento, consultas de enfermagem ou médicas, e triagem, auxiliando os profissionais e direcionando os cuidados adequados para promoção da saúde.

Além disso, o produto oportuniza a identificação de sinais e sintomas relacionados ao estresse leve, moderado ou até mesmo o esgotamento, reconhecendo as necessidades dos usuários e orientando-os quanto a necessidade de acompanhamento profissional e especializado. Com isso, contribui com as práticas de educação em saúde, além de favorecer o processo de atualização dos profissionais de saúde ao uso de aplicativos como auxílio.

Espera-se, com esse relato, fomentar o interesse para o desenvolvimento de outros aplicativos para dispositivos móveis cuja temática aborde a saúde, incluindo-se os transtornos de ordem mental, visto que são frequentemente negligenciados, de forma a favorecer a popularização de temas relacionados, contribuindo para a promoção da saúde, prevenção de doenças e redução das incapacidades. 


\section{Referências}

Alves, M. G. de M.; Chor, D.; Faerstein, E.; Lopes, C. de S. \& Werneck, G. L. (2004). Versão resumida da “job stress scale”: adaptação para o português. Rev Saúde Pública, v. 38, n. 2, p. 164-171.

Amorim, M. \& Bianco, P. (2011). Material didático em mídia digital: transposição de uma apostila do Colégio Dom Bosco para Tablet computer. Trabalho de Conclusão do Curso de Design Gráfico (TCC), do Setor de Ciências Humanas, Letras e Artes da Universidade Federal do Paraná.

Chaves, A. S. C.; Oliveira, C. G. M.; De Jesus, L. M. S.; Martins, J. L. \& Silva, V. C. da. (2018). Uso de aplicativos para dispositivos móveis no processo de educação em saúde: reflexos da contemporaneidade. Revista Humanidades e Inovação, v.5, n. 6.

Lima da Silva, J. L.; Soares, R. S.; Costa, F. S.; Ramos, D. S.; Lima, F. B. \& Teixeira, L. R. (2015). Fatores psicossociais e prevalência da síndrome de burnout entre trabalhadores de enfermagem intensivistas. Rev. Bras. Ter. Intensiva. 27(2):125-133.

GBD. (2018). Brazil Collaborators. Burden of disease in Brazil, 1990-2016: a systematic subnational analysis for the Global Burden of Disease Study. Lancet, $392: 760-75$.

George, T. P \& Decristofaro, C. (2016). Use of smartphones with undergraduate nursing students. Journal of Nursing Education, 55(7).

Goncalves, D. M.; Stein, A. T. \& Kapczinski, F. (2008). Avaliação de desempenho do Self-Reporting Questionnaire como instrumento de rastreamento psiquiátrico: um estudo comparativo com o Structured Clinical Interview for DSM-IV-TR. Cad. Saúde Pública, Rio de Janeiro, 24(2):380-390.

Heffernan, K. J. et al. (2016). Guidelines and recommendations for developing interactive eHealth apps for complex messaging in health promotion. JMIR mHealth and uHealth, 4(1).

Leiter, M. P. \& Maslach C. (2016). Latent burnout profiles: A new approach to understanding the burnout experience. BurnoutResearch, 3(1): 89-100.

Lopes, C. de. S. (2020). Como está a saúde mental dos brasileiros? A importância das coortes de nascimento para melhor compreensão do problema. Cad. Saúde Pública, Rio de Janeiro, 36(2), e00005020.

Lorenzetti, J.; Trindade, L. L.; Pires, D. E. P. de. \& Ramos, F. R. S. (2012). Tecnologia, inovação tecnológica e saúde: uma reflexão necessária. Texto Contexto Enferm, 21(2), 432-439.

Ludermir, A. B. \& Lewis, G. (2003). Informal work and common mental disorders. Soc Psychiatry Psychiatr Epidemiol. 38(9):485-9.

Mari, J. J. \& Williams, P. A. (1986). Validity study of a psychiatric screening questionnaire (SRQ-20) in primary care in the city of Sao Paulo. Br J. Psychiatry, 148:23-6.

Muraro, R. M. (2009). Os avanços tecnológicos e o futuro da humanidade: querendo ser Deus? Petrópolis. Vozes.

Neto, N. M. G., et al. (2020). Covid-19 e Tecnologia Digital: Aplicativos Móveis Disponíveis para Download em Smartphones. Texto contexto enferm. Florianópolis, 29, e20200150.

Oliveira, A. R. F. \& Alencar, M. S. M. (2017). The use of health applications for mobile devices as sources of information and education in healthcare. Rev Digit Bibliotecon. Cienc. Inf. 15:234-245.

Organização das Nações Unidas para a Educação, a Ciência e a Cultura. (2014). Diretrizes de políticas da UNESCO para a aprendizagem móvel. Brasília. http://unesdoc.unesco.org/images/0022/002277/227770por.pdf.

Rocha, S. R. da; Santana, E. B.; Silva, E. S. da; Carvalho, J. S. M. \& Carvalho, F. L. de Q. (2017). Uso de APPS para a promoção dos cuidados à saúde. STAES - III Seminário de Tecnologias Aplicadas em Educação e Saúde.

Tamayo, M. R. \& Troccoli, B. T. (2002). Exaustão emocional: relações com a percepção de suporte organizacional e com as estratégias de coping no trabalho. Estudos de Psicologia, v. 7, n. 1, p. 37-46.

Tamayo, M. R. \& Troccoli, B. T. (2009). Construção e validação fatorial da Escala de Caracterização do Burnout (ECB). Estud. psicol. 14(3): $213-221$.

Tavares, V. (2020). A saúde dos que estão na linha de frente. Escola Politécnica de Saúde Joaquim Venâncio. EPSJV/Fiocruz. http://www.epsjv.fiocruz.br/noticias/reportagem/a-saude-dos-que-estao-na-linha-de-frente.

Vigo, D.; Thornicroft, G. \& Atun, R. (2016). Estimating the true global burden of mental illness. Lancet Psychiatry, 3:171-8.

Vilela, E. M. \& Mendes, I. J. M. (2003). Interdisciplinaridade e saúde: estudo bibliográfico. Rev. Latino-Am. Enfermagem, Ribeirão Preto, 11(4):525-531.

World Health Organization. (2011). Health: new horizons for health through mobile technologies: based on the findings of the second global survey on eHealth. Geneva: WHO. (Global observatory for eHealth series, 3). 